\title{
Ectopic migration of Dirofilaria immitis in a Eurasian otter (Lutra lutra) in Korea
}

\author{
Ji-Youl Jung ${ }^{1}$, Kyunghyun Lee ${ }^{1}$, Hyun-Ji Seo ${ }^{2}$, Yun Sang Cho ${ }^{2}$, Young-Dae Kim³ , Ji-Hyeon Kim, \\ ByungJae So ${ }^{1}$, Eun-Jin Choi ${ }^{1}$ * \\ ${ }^{1}$ Animal Disease Diagnostic Division, and ${ }^{2}$ Bacterial Disease Division, Animal and Plant Quarantine Agency, \\ Gimcheon 39660, Korea \\ ${ }^{3}$ Onnury Animal Clinic, Suncheon 57949, Korea
}

(Received: January 29, 2018; Revised: April 10, 2018; Accepted: April 16, 2018)

\begin{abstract}
An 8-year-old female Eurasian otter (Lutra lutra) reared in a wetland center, died $2 \mathrm{~h}$ after sudden onset of astasia and dyspnea despite medical treatment. Gross examination of internal organs revealed 10 adult filarioid nematodes in the right ventricle of the heart and three between the left and right cerebral hemispheres. All nematodes were identified as Dirofilaria immitis by direct microscopy and polymerase chain reaction assay. Histopathological observation revealed multifocal hemorrhage in the cerebral subarachnoid space and focal necrosis with hemorrhage in the cerebellar parenchyma. Although rare, veterinarians should consider cerebral dirofilariasis as a differential diagnosis in unexplained neurological cases.
\end{abstract}

Keywords: Dirofilaria immitis, Eurasian otter, brain, ectopic migration

Dirofilariasis, caused by Dirofilaria immitis (D. immitis), exists worldwide, and has been reported from several wild and domestic canids, felids, and humans in tropical and temperate regions [11]. Mosquitoes are the most important vectors for D. immitis infection [9]. When an infected mosquito bites a susceptible animal, third-stage $D$. immitis larvae (L3) penetrate the host's skin and reach blood vessels where they develop until adulthood [9, 11]. Adult heartworms normally live in the pulmonary artery and right ventricle, but have been found in a variety of unusual sites, including eye and brain [10]. Only a few cases of $D$. immitis infection have been reported in otters, mostly in the United States of America [12], Venezuela [14], and Korea [7], and little is known about brain infection by this heartworm. The present case is the first report on the migration of adult heartworms to the brain of Eurasian otter (Lutra lutra) in Korea.

On August 7, 2017, an eight-year-old female Eurasian otter reared in a wetland center in Jeollanam-do, Korea, suddenly started to show astasia and dyspnea. During physical examination, the otter was reluctant to move. Because temperature was high (summer season), the emergency supportive care provided was based on the premise the animal was having a heat stroke. However, the otter died within $2 \mathrm{~h}$ after the onset of the clinical signs, and was submitted to the Animal Disease Diagnostic Division, Animal and Plant Quarantine Agency for postmortem examination. After necropsy, representative tissues were fixed in $10 \%$ neutral buffered formalin and stained with hematoxylin and eosin. Direct microscopy and polymerase chain reaction were performed to identify the parasite species collected.

The otter had no external abnormalities. The heart was enlarged and round, and 10 adult filarioid nematodes were observed in the right ventricle (Fig. 1A). After cutting the dura mater for macroscopic examination, three nematodes were found between the left and right cerebral hemispheres (Fig. 1B), not invading the brain parenchyma. The lungs were red and incompletely collapsed and a small amount of frothy fluid oozed from the cut surface.

Histopathological examination revealed multifocal hemorrhage in the subarachnoid space of the cerebrum and focal necrosis with hemorrhage in the cerebellar parenchyma (Fig. 1C). The lungs showed moderate congestion, edema, and myointimal proliferation of pulmonary arteries. However, no microfilaria were observed in the blood vessels of internal organs.

Thirteen filarioid nematodes observed in the heart and brain were gently removed from these organs and washed in normal saline for morphological examination of male and female characteristics as described in a previous study [6]. For the identification of parasitic species, genomic DNA was

*Corresponding author

Tel: +82-54-912-0460, Fax: +82-54-912-0465

E-mail: choiej@korea.kr 

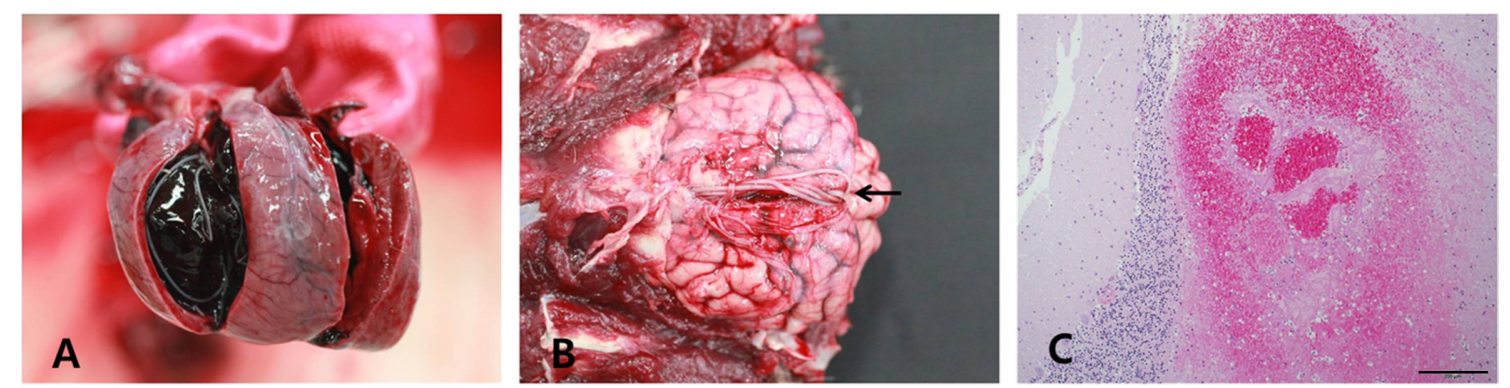

Fig. 1. Gross and histopathologic results. (A) Nematodes in the right ventricle of the heart and (B) subdural space of the brain (arrow). (C) Hemorrhage in the cerebellar parenchyma. H\&E stain. Scale bar $=200 \mu \mathrm{m}$.

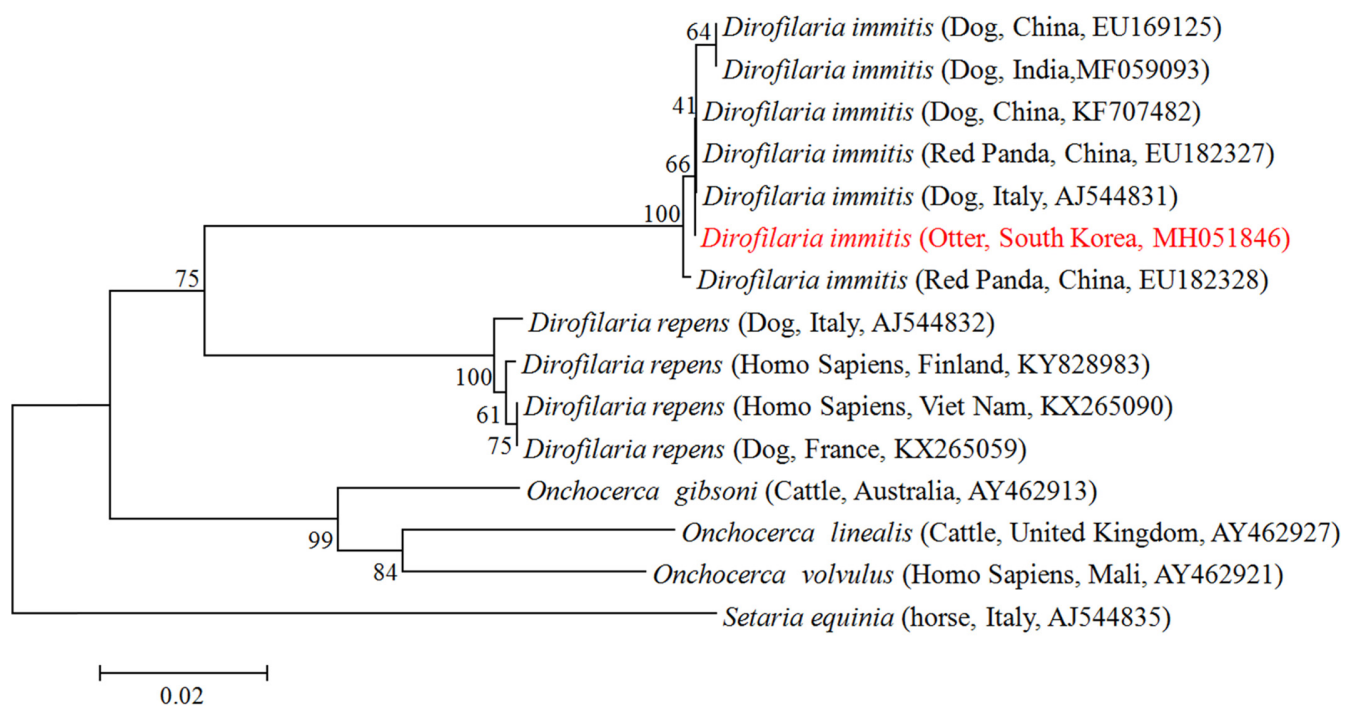

Fig. 2. Phylogenetic tree of partial $12 \mathrm{~S}$ rRNA gene sequences of filariae, nematodes, and polymerase chain reaction-amplified products in this study. The tree was generated by neighbor-joining analysis with 1,000 bootstrapped replicates. The scale bar indicates 0.02 nucleotide substitutions per site.

extracted from homogenate worms using the QIAamp DNA Mini Kit (Qiagen, Germany). Multiplex-polymerase chain reaction (PCR) was performed using $12 \mathrm{~S}$ rRNA primers previously designed for $D$. immitis and Dirofilaria repens $(D$. repens) [3]. Each amplicon was purified using QIAEX II Quick Purification Kit (Qiagen) and cloned into pGEM-T Easy Vector System I (Promega, USA). Cloned plasmid DNAs were cut by EcoRI (New England Biolabs, USA) to confirm the inserted gene and electrophoresed on an agarose gel $(1.2 \%)$. Nucleotide sequences obtained by Macrogen (Korea) were compared with corresponding 12S rRNA sequences without the primer region deposited in the National Center for Biotechnology Information GenBank database (USA) using the multisequence alignment program of MegAlign (ver. 7.1; DNASTAR, USA). Phylogenetic trees were generated using neighbor-joining algorithms. Support for topology was calculated using 1,000 bootstrap replications.

Among the 13 nematodes examined, 10 from the heart and three from the brain, nine were females and four were males. Females were $22.1-24.0 \mathrm{~cm}$ and males were $13.2-14.3 \mathrm{~cm}$ in length. Males had a spirally coiled posterior end whereas females were straight on both ends. The amplicons obtained by the multiplex-PCR reaction performed for all nematodes showed an expected size of approximately $500 \mathrm{bp}$ for the genus Dirofilaria and 204 bp for D. immitis. The consensus 12S rRNA DNA sequence was deposited in GenBank under accession No. MH051846. In the phylogenetic tree, MH051846 was clustered with various $D$. immitis (Fig. 2), and all sequences analyzed showed $99.8-100 \%$ similarity to $D$. immitis. The highest similarity $(100 \%)$ was obtained toward AJ544831, KF707482, and EU182327.

Based on the pathological features and parasitological findings, this case was diagnosed as a $D$. immitis infection. The genus Dirofilaria includes 40 recognized species, but veterinary concern has recently focused on $D$. immitis and $D$. repens. Despite the continuous reports of dirofilariasis, there is still little information on this disease in wild animals. The single case of dirofilariasis in a Eurasian otter was reported in Korea [7], but there is no record on the ectopic migration of $D$. immitis in this species worldwide.

Although $D$. immitis are usually found in the right side of the heart or in pulmonary arteries, these heartworms have 
been observed in other sites including the venae cavae, hepatic veins, bronchioles, eye, peritoneal cavity, aorta, femoral arteries, and brain [5]. In the present case, three D. immitis were observed in the subdural space of the brain. Moreover, this otter showed neurological symptoms such as astasia and hemorrhage in the subarachnoid space and cerebellum. Although there were no D. immitis in the brain parenchyma, the migration to the brain itself might have led to the clinical signs displayed by the otter.

The final maturation of juvenile worms to adult heartworms occurs in the pulmonary vessels [8]. Live heartworms can cause villous endarteritis and sclerosis of the intima and media in the vessels [8]. However, in this case, there were no lesions in the brain vessels such as endarteritis. Only mild subdural hemorrhage and focal cerebellar necrosis were observed. Moreover, the otter showed sudden onset of neurological symptoms. These results suggest that adult heartworms migrated to the brain rather than maturing there.

D. immitis infection can be diagnosed through careful morphological examination of circulating microfilariae, detection of circulating antigens, histochemical or immuno-histochemical staining of circulating microfilariae or, more recently, through molecular approaches [2]. Diagnostic tests using genomic characteristics are increasingly used to identify Dirofilaria species infecting hosts. In the present case, infection by $D$. immitis was confirmed by direct microscopy and PCR.

Most species of otters live in and/or close to water but they spend much time on land, where they are exposed to mosquito bites. $D$. immitis is transmitted by culicid mosquito species belonging to a wide range of genera including Culex, Aedes, Ochlerotatus, Anopheles, Armigeres, and Mansonia [1]. Anopheles sinensis, Aedes vexans nipponii, and Culex pipiens were referred as potential vectors of $D$. immitis in Korea [4].

Dirofilariasis is an important parasitic disease in animals. Moreover, increasing rates of $D$. immitis infection have been associated with climate change [11]. Global warming and its impact on the spread of vector-borne parasites is a major concern, and Korea, although located in a temperate zone, has favorable conditions for the spread of such parasites during the hot and humid summer season [13]. Early diagnosis and control of these pathogens in domestic animals and wildlife are thus needed.

This case seems to be the first report on the ectopic migration of $D$. immitis to the brain of a Eurasian otter in Korea. Although quite rare, veterinarians should consider cerebral dirofilariasis as a differential diagnosis in any unexplained neurological case.

\section{Acknowledgments}

This work was supported financially by a grant $(\mathrm{N}-$ 1543069-2015-99-01) from the Animal and Plant Quarantine Agency, Republic of Korea.

\section{References}

1. Cancrini G, Pietrobelli M, Frangipane di Regalbono AF, Tampieri MP, della Torre A. Development of Dirofilaria and Setaria nematodes in Aedes albopictus. Parassitologia 1995, 37, 141-145.

2. Ceribasi AO, Simsek S. Histopathologic effects of Dirofilaria immitis microfilaria on internal organs of dog confirming by PCR technique. Iran J Parasitol 2012, 7, 103-107.

3. Gioia G, Lecová L, Genchi M, Ferri E, Genchi C, Mortarino M. Highly sensitive multiplex PCR for simultaneous detection and discrimination of Dirofilaria immitis and Dirofilaria repens in canine peripheral blood. Vet Parasitol 2010, 172, 160-163.

4. Lee SE, Kim HC, Chong ST, Klein TA, Lee WJ. Molecular survey of Dirofilaria immitis and Dirofilaria repens by direct PCR for wild caught mosquitoes in the Republic of Korea. Vet Parasitol. 2007, 148, 149-155.

5. Liu SK, Das KM, Tashjian RJ. Adult Dirofilaria immitis in the arterial system of a dog. J Am Vet Med Assoc 1966, 148, 1501-1507.

6. Manfredi MT, Di Cerbo A, Genchi M. Biology of filarial worms parasitizing dogs and cats. In: Genchi C, Rinaldi L, Cringoli G (eds.). Mappe Parassitologiche. Dirofilaria immitis and D. repens in Dog and Cat and Human Infections. Vol. 8. pp. 41-45, Rolando Editore, Naples, 2007.

7. Matsuda K, Baek BK, Lim CW. Eurasian otter (Lutra lutra), a definitive host for Dirofilaria immitis. J Zoo Wildl Med 2003, 34, 200-201.

8. Nelson CT. Heartworm disease. In: Greene CE (ed.). Infectious Diseases of the Dog and Cat. 4th ed. pp. 865-876. Saunders, Philadelphia. 2011.

9. Oh IY, Kim KT, Sung HJ. Molecular detection of Dirofilaria immitis specific gene from infected dog blood sample using polymerase chain reaction. Iran J Parasitol 2017, 12, 433-440.

10. Robinson WF, Robinson NA. Cardiovascular system. In: Maxie MG (ed.). Jubb, Kennedy, and Palmer's Pathology of Domestic Animals. Vol. 3. 6th ed. pp. 83-85, Elsevier, St. Louis, 2016.

11. Simón F, Siles-Lucas M, Morchón R, González-Miguel J, Mellado I, Carretón E, Montoya-Alonso JA. Human and animal dirofilariasis: the emergence of a zoonotic mosaic. Clin Microbiol Rev 2012, 25, 507-544.

12. Snyder DE, Hamir AN, Nettles VF, Rupprecht CE. Dirofilaria immitis in a river otter (Lutra Canadensis) from Louisiana. J Wildl Dis 1989, 25, 629.

13. Song KH, Lee SE, Hayasaki M, Shiramizu K, Kim DH, Cho KW. Seroprevalence of canine dirofilariasis in South Korea. Vet Parasitol 2003, 114, 231-236.

14. Vogelsang EG. [Parasites of animals in Venezuela. XIII. Hosts of D. immitis in Venezuela]. Rev Med Vet Parasitol 1940, 2, 91-93. Spanish. 